\title{
BMJ Open Effectiveness of blood pressure- lowering drug treatment by levels of absolute risk: post hoc analysis of the Australian National Blood Pressure Study
}

Chau Le Bao Ho, ${ }^{1}$ Monique Breslin, ${ }^{1}$ Jenny Doust, ${ }^{2}$ Christopher M Reid, ${ }^{3,4}$ Mark R Nelson ${ }^{1,4}$

To cite: Ho CLB, Breslin M, Doust J, et al. Effectiveness of blood pressure-lowering drug treatment by levels of absolute risk: post hoc analysis of the Australian National Blood Pressure Study. BMJ Open 2018;8:e017723. doi:10.1136/ bmjopen-2017-017723

- Prepublication history and additional material for this paper are available online. To view these files, please visit the journal online (http://dx.doi. org/10.1136/bmjopen-2017017723).

Received 12 May 2017 Revised 7 December 2017 Accepted 30 January 2018

\section{Check for updates}

${ }^{1}$ Menzies Institute for Medical Research, University of Tasmania, Hobart, Tasmania, Australia

${ }^{2}$ Faculty of Health Sciences and Medicine, Bond University, Gold Coast, Queensland, Australia

${ }^{3}$ School of Public Health, Curtin University, Perth, Western Australia, Australia

${ }^{4}$ CCRE Therapeutics, School of Public Health and Preventive Medicine, Monash University, Melbourne, Victoria, Australia

Correspondence to

Dr Chau Le Bao Ho;

chau.ho@utas.edu.au

\section{ABSTRACT}

Objectives In many current guidelines, blood pressure (BP)-lowering drug treatment for primary prevention of cardiovascular disease (CVD) is based on absolute risk. However, in clinical practice, therapeutic decisions are often based on BP levels alone. We sought to investigate which approach was superior by conducting a post hoc analysis of the Australian National Blood Pressure (ANBP) cohort, a seminal study establishing the efficacy of BP lowering in 'mild hypertensive' persons.

Design A post hoc subgroup analysis of the ANBP trial results by baseline absolute risk tertile.

Setting and participants 3244 participants aged 35-69 years in a community-based randomised placebo controlled trial of blood pressure-lowering medication.

Interventions Chlorothiazide500 mg versus placebo. Primary outcome measures All-cause mortality and nonfatal events (non-fatal CVD, congestive cardiac failure, renal failure, hypertensive retinopathy or encephalopathy). Results Treatment effects were assessed by HR, absolute risk reduction and number needed to treat. Participants had an average 5-year CVD risk in the intermediate range $(10.5 \pm 6.5)$ with moderately elevated $\mathrm{BP}$ (mean $159 / 103 \mathrm{mmHg}$ ) and were middle aged ( $52 \pm 8$ years). In a subgroup analysis, the relative effects (HR) and absolute effects (absolute risk reduction and number needed to treat) did not statistically differ across the three risk groups except for the absolute benefit in all-cause mortality ( $p$ for heterogeneity=0.04). With respect to absolute benefit, drug treatment significantly reduced the number of events in the high-risk group regarding any event with a number needed to treat of 18 (10 to 64), death from any cause with 45 (25 to 196) and major CVD events with 23 (12 to 193).

Conclusion Our analysis confirms that the benefit of treatment was substantial only in the high-risk tertile, reaffirming the rationale of treating elevated blood pressure in the setting of all risk factors rather than in isolation.

\section{INTRODUCTION}

For decades, cardiovascular disease (CVD) has remained the greatest burden of disease in the developed world and now also in the
Strengths and limitations of this study

- Our analysis provides further justification that an absolute risk strategy is superior to management based on the BP level alone in identifying those who are most likely to benefit from therapy.

- The statistical power to detect treatment effects was limited in this study, and this is a post hoc subgroup analysis.

- Due to the lack of high-density lipoprotein cholesterol (HDLC) in the original data set, the HDLc used in the analyses was imputed from a 1980s national survey. The use of these imputed values is unlikely to greatly affect the risk stratification.

developing world. ${ }^{12}$ In 2012, CVD was responsible for 17.5 million deaths in the world and more than 20000 deaths in Australia. ${ }^{13}$ Noticeably, nearly $50 \%$ of deaths from CVD are attributable to high blood pressure (BP), the most common modifiable population risk factor. ${ }^{4}$ Drug therapy for primary prevention of CVD is now recommended to be based on absolute CVD risk, where BP-lowering drug treatment is determined by BP level together with other major CVD risk factors (eg, sex, age, total cholesterol, high-density lipoprotein cholesterol (HDLc), diabetes and smoking status) as an integrated score.$^{5-9}$ Yet clinicians are reticent to treat systolic BP in those below $140 \mathrm{mmHg}$ at high risk as well as not treating patients at low risk with $\mathrm{BP}$ above this threshold. There is a paucity of literature on the effects of lowering BP in low to moderate CVD risk individuals with grade 1 hypertension (systolic BP from $140 \mathrm{mmHg}$ to $159 \mathrm{mmHg}$ and/or diastolic BP from $90 \mathrm{mmHg}$ to $99 \mathrm{mmHg}$ ) and some debate regarding its benefit. ${ }^{10}$ Guidelines from the US and Europe focus on BP thresholds and 
promote early drug treatment due to the potential benefits of earlier intervention and potential adverse effects of delayed intervention. ${ }^{6-8}{ }^{11-13} \mathrm{JNC}^{11}$ recommends initiating drug treatment at the threshold of $150 \mathrm{mmHg}$ systolic BP or $90 \mathrm{mmHg}$ diastolic BP for the general population at 60 years or older. This revised recommendation has caused controversy among clinicians who argue that drug treatments need to be initiated at a lower systolic $\mathrm{BP}$ of $140 \mathrm{mmHg}$, as previously recommended in JNC $7^{14}$; otherwise, patients are exposed to increased risk. ${ }^{15-18}$ Similarly, the 2016 European Society of Cardiology guidelines recommend considering BP-lowering drug treatment when systolic BP is greater than $140 \mathrm{mmHg}$ and/or diastolic BP is greater than $90 \mathrm{mmHg}$ after a reasonable period of time with lifestyle choice. ${ }^{7}$ Recently, the Systolic Blood Pressure Intervention (SPRINT) trial ${ }^{19}$ reported a significant benefit from intensive treatment to a target BP of $120 \mathrm{mmHg}$ rather than $140 \mathrm{mmHg}$. However, this benefit was observed in those at high CVD risk without diabetes. In agreement with the findings from the SPRINT trial, guidelines in Australia, ${ }^{5}$ New Zealand, ${ }^{20}$ $\mathrm{UK}^{8}$ and Canada ${ }^{9}$ recommend BP-lowering medication based on absolute CVD risk, recommending BP-lowering treatment as soon as possible in high CVD risk individuals, but not in the low-risk to moderate-risk population unless BP persistently exceeds $160 / 100 \mathrm{mmHg}$.

Other groups ${ }^{21}$ have recommended early drug treatment of grade 1 hypertension even in patients at low risk with the exception of patients with grade 1 'isolated' hypertension, based on a meta-analysis by Thomopoulos et $a l^{22}$ and the HOPE-3 study. ${ }^{23}$ In contrast, a Cochrane review by Diao et $a l^{10}$ concluded that there was no statistically significant effect of BP treatment in individuals who had grade 1 hypertension. The 2015 Blood Pressure Lowering Treatment Trialists Collaboration ${ }^{24}$ (BPLTTC) meta-analysis reported a statistically significant benefit of BP-lowering drug treatment in grade 1 hypertension in terms of stroke and all-cause mortality. However, the effects seen in the BPLTTC analysis could reflect differences in the BPLTTC sample that included participants who had diabetes, had a higher baseline risk and had previously received drug treatment. In another analysis of the BPLTTC individual patient data ${ }^{25}$ by absolute CVD risk at baseline showed a continuously increasing benefit with baseline risk. ${ }^{25}$ The BPLTTC study, however, included participants who both did and did not have a history of CVD.

Thus, we sought to reanalyse a seminal study used to justify treating individuals with elevated BP to see if stratification by baseline CVD risk would be a superior method for identifying candidates for BP-lowering medication in a treatment-naive population. In this study, we compared the effectiveness of BP-lowering drug treatment by a post hoc subgroup analysis of the Australian National Blood Pressure (ANBP) study. ${ }^{26}$ We restricted the analysis group to individuals with no history of CVD or diabetes and who were naive to BP-lowering treatment. We selected this historical study because it was placebo controlled and patients in the control arm of the study would not have been taking a BP-lowering medication previously unless they had very high levels of BP. Our aim was to assess which group of individuals classified by absolute risk benefited from active treatment versus placebo for CVD events within this seminal study that underwrote the treatment of elevated BP by BP thresholds.

\section{METHODS}

We performed a post hoc analysis of the ANBP study ${ }^{26}$. The study was conducted between 1973 and 1979 and was a multicentre, single-blind randomised controlled trial of 3427 patients that compared the effects of BP-lowering drug therapy between individuals who initially received active treatment (chlorothiazide) and those who received delayed active treatment or no active treatment (placebo). The study intervention remains applicable to current practice as thiazide diuretics (eg, hydrochlorothiazide) are still first-line BP-lowering agents. ${ }^{5-9}$ The ANBP study enrolled participants who had not been on treatment for hypertension in the past 3 months and had no history of CVD or diabetes. In the 1970s, 'mild hypertension' was defined as a screening diastolic BP of $95-109 \mathrm{mmHg}$ with a systolic BP lower than $200 \mathrm{mmHg}$. A total of 3931 eligible participants were initially randomised, then 504 participants were excluded because their BP throughout the study did not meet the criteria for starting drug treatment (entry or follow-up diastolic BP higher than $95 \mathrm{mmHg}$ and/or entry or follow-up systolic BP higher than $200 \mathrm{mmHg}$ ). The primary endpoints were all-cause mortality and non-fatal events (non-fatal CVD, congestive cardiac failure, renal failure, hypertensive retinopathy or encephalopathy). ${ }^{26}$

\section{Risk stratification}

In this analysis, the baseline absolute CVD risk was calculated according to the 5-year Framingham absolute risk score. ${ }^{27}$ The Framingham score was chosen because it is currently recommended in the National Vascular Disease Prevention Alliance (NVDPA) guidelines ${ }^{5}$ in Australia. The sample was restricted to 3244 participants who were older than 35 years and was stratified by tertile of estimated 5-year CVD risk score. We also classified participants with very high BP (systolic BP $\geq 180 \mathrm{mmHg}$ and/or diastolic $\mathrm{BP} \geq 110 \mathrm{mmHg})$ or total cholesterol $(>7.5 \mathrm{mmol} / \mathrm{L})$ values the highest risk tertile regardless of their risk score, as per the Australian guidelines. ${ }^{5}$ The ANBP dataset included all variables required for CVD risk calculation except HDLc. The HDLc value was imputed from the Australian National Heart Foundation risk factor prevalence study as this was near contemporaneous with the ANBP. ${ }^{28}$ Mean value of HDLc was categorised by age and sex. In a sensitivity analysis, we stratified the sample by Globorisk score,${ }^{29}$ a CVD risk score that does not require HDLc value and is validated in individuals over 40 years. The equation for the Australian population was obtained by personal contact with the author (Peter Ueda, unpublished data, 2016). This analysis excluded 471 participants younger than 40 years. Less 
than $1 \%$ of the study participants had data missing for total cholesterol, weight and/or height, and these missing data were managed by multiple imputation using chained equations.

\section{Statistical analysis}

All analyses were based on the modified 'intention to treat' principle. We included participants who had withdrawn from the study by their group allocation at randomisation in all analyses. The differences in baseline characteristics between 'active group' and 'placebo group' were tested by analysis of variance test for continuous variables and $\chi^{2}$ test for categorical variables. Treatment effects were assessed by $\mathrm{HR}$, absolute risk reduction (ARR) and number needed to treat (NNT). The HRs and corresponding 95\% CIs were estimated by Cox proportional hazard model after adjusting for clustering of participants within community-based centres and potential risk factors including baseline characteristics. The proportional assumption was checked by the test for interaction of HR with time. ARR and NNT were estimated from Kaplan-Meier curves at the median of follow-up time (4.4 years). ${ }^{30}$ Tests for interaction of treatment effect over the subgroups were obtained by the Cox regression model for the HR and a Cochran's $Q$ test for the ARR. The threshold for significance for treatment effect was set at 0.05 for the main analysis and subgroup analysis. Only one subgroup analysis with related outcomes was conducted; thus, multiplicity was not likely to affect our results.

\section{RESULTS}

\section{Patient characteristics}

Table 1 provides baseline characteristics of the participants stratified by the tertile of CVD risk score. On average, study participants had intermediate 5-year CVD risk as referred in the NVDPA guideline $(10.5 \pm 6.5)$ with moderately elevated BP (mean $159 / 103 \mathrm{mmHg}$ ) and were middle aged $(52 \pm 8)$. The tertiles had estimated 5-year CVD risks of less than $6.1 \%$ (low), $6.1 \%-17.0 \%$ (moderate) and more than $17.0 \%$ (high). These values are similar to the thresholds recommended by the Australian NVDPA guideline ${ }^{5}$ for low $(<10 \%)$, moderate $(10 \%-15 \%)$ and high risk categorisation $(>15 \%)$. The distribution of baseline characteristics by treatment assignment was not significantly different except for body mass index (BMI) in the total population, the number of smokers in the low-risk group, systolic BP and BMI in the moderate-risk group.

Approximately one-third of the participants (34.5\%) prematurely stopped study treatment due to decisions by clinics, participants' doctors and the participant themselves, or for unknown reasons (table 2). Participants' doctors were more likely to stop placebo treatment in all three risk groups, whereas clinics withdrew more BP-lowering drug-randomised participants in the low-risk group and the high-risk group. No substantial difference in baseline characteristics between the two randomised treatment groups was recorded in any risk group.

\section{Effect of BP-lowering drug treatment on total study population} During a median follow-up of 4.4 years (IQR 1.0-5.9), 257 major CVD events $(7.9 \%)$ were observed, in which ischaemic heart disease accounted for 203 events $(6.3 \%)$, stroke accounted for 48 events $(1.5 \%)$ and congestive heart failure accounted for six events $(0.2 \%)$.

After adjustment for sex, age, BMI, smoking, systolic BP at baseline and study centres, BP-lowering treatment was associated with a $15 \%$ reduction in non-fatal events and a $25 \%$ reduction in all-cause mortality (figure 1 ), although the treatment effects were not statistically significant. Similar effects were found in the secondary endpoints including any events HR 0.82 (0.65-1.03), major CVD events HR 0.83 (0.65-1.07) and non-fatal CVD events HR $0.87(0.67-1.13)$. We identified a marginally significant effect in stroke HR 0.55 (0.3-1.001).

\section{Effect of BP-lowering drug treatment on 5-year CVD risk groups}

In the subgroup analysis, the magnitude of relative treatment effect increased from low to high CVD risk group,

\begin{tabular}{|c|c|c|c|c|}
\hline Group variable & Total & $\begin{array}{l}\text { Low } \\
(<6.1 \%)\end{array}$ & $\begin{array}{l}\text { Moderate } \\
(6.1 \%-17.0 \%)\end{array}$ & $\begin{array}{l}\text { High } \\
(>17.0 \%)\end{array}$ \\
\hline Sample, N & 3244 & 1082 & 1081 & 1081 \\
\hline Randomised to active treatment, $\mathrm{N}(\%)$ & $1622(50 \%)$ & $559(51.7 \%)$ & $513(47.5)$ & $550(50.9)$ \\
\hline Age, years & $51.7 \pm 8.1$ & $46.0 \pm 6.2$ & $54.5 \pm 6.5$ & $54.6 \pm 8.1$ \\
\hline Male sex, N (\%) & 2017 (62.2) & $567(52.4)$ & $804(74.4)$ & $646(59.8)$ \\
\hline Current smoker, $\mathrm{N}(\%)$ & $801(24.7)$ & $115(10.6)$ & $352(32.6)$ & $334(30.9)$ \\
\hline SBP, mmHg & $159.5 \pm 17.5$ & $148.4 \pm 12.2$ & $157.3 \pm 12.2$ & $172.6 \pm 17.9$ \\
\hline DBP, $\mathrm{mmHg}$ & $102.9 \pm 6.8$ & $100.0 \pm 3.8$ & $100.8 \pm 4.4$ & $107.9 \pm 8.2$ \\
\hline Total cholesterol, $\mathrm{mmol} / \mathrm{L}$ & $6.0 \pm 1.1$ & $5.5 \pm 0.9$ & $6.0 \pm 0.9$ & $6.5 \pm 1.3$ \\
\hline $\mathrm{BMI}, \mathrm{kg} / \mathrm{m}^{2}$ & $26.6 \pm 3.9$ & $26.6 \pm 4.0$ & $26.5 \pm 3.6$ & $26.7 \pm 4.1$ \\
\hline
\end{tabular}

Bold values: $\mathrm{P}<0.05$ based on the distribution of baseline characteristics by treatment assignment.

$\mathrm{BMI}$, body mass index; CVD, cardiovascular disease; DBP, diastolic blood pressure; SBP, systolic blood pressure. 
Table 2 Characteristics of those who prematurely stopped study regimen

\begin{tabular}{|c|c|c|c|c|}
\hline Group variable & Total & $\begin{array}{l}\text { Low } \\
(<6.1 \%)\end{array}$ & $\begin{array}{l}\text { Moderate } \\
(6.1 \%-17.0 \%)\end{array}$ & $\begin{array}{l}\text { High } \\
(>17.0 \%)\end{array}$ \\
\hline Sample, N & 1119 & 404 & 346 & 369 \\
\hline Randomised to active treatment, N (\%) & $531(47.5)$ & $204(50.5)$ & $151(43.6)$ & $176(47.7)$ \\
\hline Age, years & $51.2 \pm 8.3$ & $45.9 \pm 6.4$ & $54.1 \pm 7.0$ & $54.2 \pm 8.5$ \\
\hline Male sex, N (\%) & $626(55.9)$ & $188(46.5)$ & $243(70.2)$ & $195(52.9)$ \\
\hline Current smoker, $\mathrm{N}(\%)$ & $321(28.7)$ & $58(14.4)$ & $143(41.3)$ & $120(32.5)$ \\
\hline SBP, $\mathrm{mmHg}$ & $159.1 \pm 18.1$ & $147.6 \pm 12.9$ & $157.0 \pm 11.9$ & $173.7 \pm 17.7$ \\
\hline DBP, $\mathrm{mmHg}$ & $102.9 \pm 6.8$ & $100.0 \pm 4.0$ & $100.6 \pm 4.2$ & $108.1 \pm 8.2$ \\
\hline Total cholesterol, mmol/L & $6.0 \pm 1.1$ & $5.5 \pm 0.9$ & $6.0 \pm 0.9$ & $6.4 \pm 1.3$ \\
\hline $\mathrm{BMI}, \mathrm{kg} / \mathrm{m}^{2}$ & $26.7 \pm 4.1$ & $26.7 \pm 4.0$ & $26.6 \pm 3.9$ & $26.8 \pm 4.5$ \\
\hline \multicolumn{5}{|l|}{ Reason for stopping } \\
\hline Clinic, N (\%) & $204(18.2)$ & 74 (18.3) & 75 (21.7) & 55 (14.9) \\
\hline Local doctor, N (\%) & $287(25.7)$ & $98(24.3)$ & $87(25.1)$ & $102(27.6)$ \\
\hline Participants, N (\%) & $548(49.0)$ & 204 (50.5) & $162(46.8)$ & $182(49.3)$ \\
\hline Not known, N (\%) & $80(7.2)$ & $28(6.9)$ & $22(6.4)$ & $30(8.1)$ \\
\hline
\end{tabular}

Bold values: $\mathrm{P}<0.05$ based on the distribution of baseline characteristics by treatment assignment.

$\mathrm{BMI}$, body mass index; CVD, cardiovascular disease; DBP, diastolic blood pressure; SBP, systolic blood pressure.

though the benefits were not statistically significant in the high-risk group in terms of all-cause mortality $0.60(0.26-$ $1.40)$ and major CVD event with HR $0.76(0.52-1.10)$.

The increasing trend for the benefit was also observed when comparing the absolute treatment effects ARR among the three risk groups. No evidence of heterogeneity was observed except the effect in the major CVD event. Substantial effects of BP-lowering treatment were produced in the high-risk group regarding any trial endpoints (ARR 5.6 (95\% CI 1.6 to 9.6)), all-cause mortality (ARR 2.2 (95\% CI 0.5 to 3.9 ) ) and any CVD event (4.3 (95\% CI 0.5 to 8.1)) (table 3). Treating 18 high-risk participants for 4 years prevented one trial event, treating 45 prevented one death and treating 23 prevented one CVD event. In contrast, treating low or moderate-risk participants needed much higher numbers to prevent one event or possibly caused net harm (table 3). Also, a sensitivity analysis by using the GLOBORISK score, ${ }^{29}$ which does not require HDLc, was consistent with our original findings, except that the ARR in major CVD event is no longer statistically significant with ARR 3.4\% $(-0.4 \%$ to $7.3 \%, \mathrm{P}=0.08)$.

\section{DISCUSSION}

In our post hoc analysis of the ANBP study, we found evidence of benefit from BP-lowering treatment in the high-risk tertile for primary trial endpoints of any event and any CVD event with low or moderate-risk participants unlikely to benefit. Our study population had an overall moderate 5-year CVD risk $(10.5 \%)$ and moderately elevated systolic BP (mean $159 / 103 \mathrm{mmHg}$ ) by modern definitions. The ANBP study aimed to treat 'mild hypertension' (according to the old definition) that
Any event

All-cause mortality

Non-fatal event

Major CVD event

Stroke

CVD mortality

Any CHD

\begin{tabular}{ll}
\multicolumn{2}{c}{$\begin{array}{r}\text { Event (rate per } 1000 \\
\text { patient-yr) }\end{array}$} \\
Active & Placebo \\
$137(19.6)$ & $165(23.8)$ \\
$25(3.4)$ & $33(4.5)$ \\
$112(13.2)$ & $132(15.9)$ \\
$117(16.7)$ & $140(20.0)$ \\
$17(2.3)$ & $31(4.3)$ \\
$8(1.1)$ & $15(2.0)$ \\
$97(13.7)$ & $106(15.0)$
\end{tabular}

Adjusted HR $(95 \% \mathrm{Cl})^{*}$

$0.82(0.65,1.03)$

$0.75(0.45,1.36)$

$0.85(0.66,1.09)$

$0.83(0.65,1.07)$

$0.55(0.31,1.001)$

$0.56(0.23,1.31)$

$0.92(0.70,1.22)$

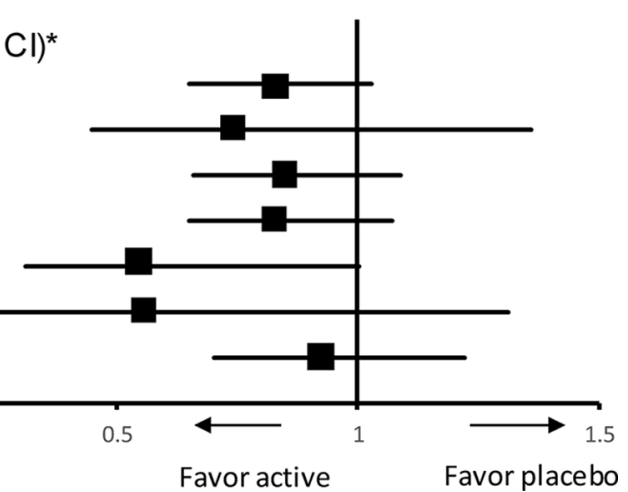

Figure 1 Effect of treatment in the overall study population. *Adjusted for age, sex, body mass index, screening centres, smoking and systolic blood pressure. Bold values: $\mathrm{P}<0.05$. CHD, coronary heart disease; CVD, cardiovascular disease. 
Table 3 Effect of treatment by tertile of baseline CVD risk score

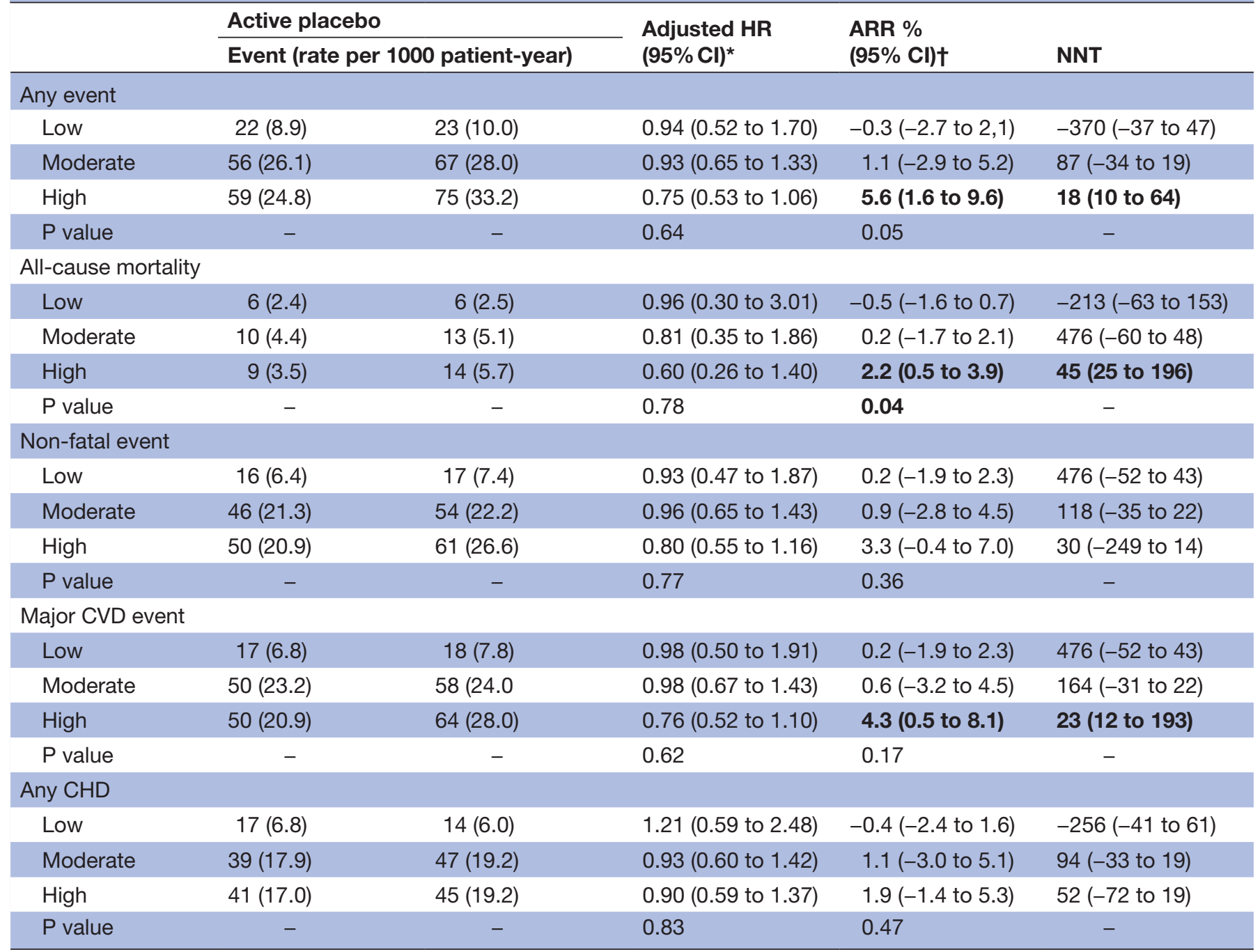

Bold values: $\mathrm{P}<0.05$.

$P$ value indicated $p$ for interaction.

${ }^{*}$ Adjusted for age, sex, body mass index, smoking, screening centres and systolic blood pressure.

†As estimated by the Kaplan-Meier curve.

ARR, absolute risk reduction; CHD, coronary heart disease; CVD, cardiovascular disease; NNT, number needed to treat; NNTB, NNT (benefit); NNTH, NNT (harm).

was primarily defined by diastolic BP. Some randomised participants were excluded from the original analysis because they did not meet the criteria for starting BP-lowering drug treatment postrandomisation. This would not be seen in modern clinical trials. In our reanalysis, we found that BP-lowering drug treatment reduced the risk of major CVD events and all-cause mortality, but the effect was not statistically significant. This is likely to be due to reduced power as the cohort was analysed by tertile of absolute risk, as well as by the two groups of randomised therapy. The original study found a statistically significant reduction in the incidence of CVD mortality and all trial endpoints, using the full dataset and a risk ratio (RR) rather than time-to-event analysis. ${ }^{26}$

In our analysis of subgroups defined by CVD risk score, the magnitude of relative treatment effects (relative risk reduction) on all-cause mortality and major CVD events increased across all three CVD risk group from low to high risk, without statistically significant heterogeneity ( $\mathrm{P}=0.78$ for all-cause mortality and $\mathrm{P}=0.62$ for the major CVD event) (table 3). All relative treatment effects in our analysis measured by HRs were adjusted by age, sex, BMI, smoking, screening centres and systolic BP. However, no significant difference was observed between adjusted and unadjusted HRs. In terms of absolute benefits, risk reduction linearly increased across the CVD risk group from low to high risk. BP-lowering drug treatment produced an unclear benefit in the low and intermediate CVD risk group but a significant benefit in the high CVD risk group. Heterogeneity of absolute effects across the CVD risk groups was only significant in all-cause mortality $(\mathrm{P}=0.04)$.

Regarding the benefit of BP-lowering drug treatment in the low to intermediate CVD risk population, our results 
from main and subgroup analyses match well with the study outcomes from the HOPE-3 trial ${ }^{23}$ and the Diao review. ${ }^{10}$ In the HOPE-3 trial, ${ }^{23}$ no benefit of intensive drug treatment was established in the intermediate-risk persons with HR $0.98(0.84-1.14)$ for all-cause mortality and HR 0.92 (0.79-1.06) for major CVD events referred as a first secondary outcome in the paper. At baseline, the HOPE-3 participants were older (65 years) and had a lower level of BP $(138.1 / 81.9 \mathrm{mmHg})$ compared with the ANBP participants. One reason for the lower BPs may be due to the 4-week run-in phase in which all of the HOPE-3 participants received active BP-lowering drug treatment before randomisation, and one-fifth of all eligible participants had previously received drug treatment before the trial. In 2012, Diao et al reviewed placebo randomised controlled trials in grade 1 hypertension and also found no beneficial effect of drug treatment with a RR $0.85(0.63-1.15)$ for all-cause mortality and RR 0.97 $(0.2-1.32)$ for major CVD events. ${ }^{10}$ The participants in the Diao review were likely to have a lower CVD risk than those in the ANBP and the HOPE-3 trials, with major CVD events occurring in only $2.4 \%$ of participants in the placebo group. Following a similar approach, in 2015, the BPLTTC ${ }^{24}$ reviewed randomised controlled trials in grade 1 hypertension but extended to trials comparing active or more intensive regimens and placebo or less intensive regimens. In line with the findings from the 2015 BPLTTC study, we identified a marginally significant effect on stroke, yet our effect estimates with an HR $0.75(0.45-1.36)$ for total deaths and an HR 0.83 $(0.65-1.07)$ for major CVD events slightly differed from the 2015 BPLTTC study's results with an OR 0.78 (0.67$0.92)$ and an OR 0.86 (0.74-1.01) correspondingly. The differences in CIs may be due to the difference in sample sizes and baseline characteristics. It is more likely that the 2015 BPLTTC participants had higher CVD risk and higher BP value at baseline when about $40 \%$ of 15266 participants had diabetes and about $23 \%$ had previously received BP-lowering drug treatment. Our study and the 2015 review confirm the absolute benefits of BP-lowering drug treatment in high CVD risk population in terms of total deaths with ARR 2.2\% (0.5\% to $3.9 \%, \mathrm{P}=0.01)$ for the ANBP and ARR $1.4 \%$ (0.5\% to 2.2\%) for the review. Furthermore, the benefit was also recorded in major CVD event with ARR $4.3 \%$ (0.5\% to $8.1 \%, \mathrm{P}=0.03)$ in the ANBP, whereas the 2015 BPLTTC observed a non-significant effect with ARR $1.0 \%$ ( $-0.1 \%$ to $1.9 \%)$. The difference can be explained in part by the study design when more than $50 \%$ of participants with systolic BP higher than $160 \mathrm{mmHg}$ in eligible studies in the 2015 BPLTTC were excluded. The distribution of these excluded participants might not be even between active arm and control arm, thus biasing the treatment effects.

In another subgroup analysis stratified by tertile of baseline systolic BP (online supplement), the mean value of CVD risk varied from low to high corresponding to the lowest and the highest tertile. The relative treatment benefits were not statistically significant, but in terms of absolute effects, BP-lowering drug treatment substantially reduced any trial events, all-cause mortality and major CVD events within the highest tertile. The findings were in line with what we found in the CVD risk-stratified subgroup when all participants in the highest BP-stratified tertile had high CVD risk score $(20.7 \pm 9.5)$. However, the heterogeneity of treatment effects among the three subgroups in analysis by baseline systolic $\mathrm{BP}$ was no longer significant as it was in the subgroup analysis by CVD risk score. Furthermore, the trend of lower to higher absolute benefit from low-risk to high-risk groups that was seen for CVD risk was not apparent when groups are defined by BP alone. Thus, in this study, CVD risk score identified those who most benefited from BP-lowering drug treatment.

\section{Limitations}

There are a number of limitations of our study. First, statistical power is unavoidably decreased in a post hoc subgroup analysis, and the multivariate Framingham risk score used in our analysis has not been well validated within the Australian population. ${ }^{31}$ However, using a multivariate score for stratification is known to increase the power to detect heterogeneity in absolute risk benefit over subgroup analyses that are based on individual risk factors. ${ }^{32}$ A prospective study to address the issue of whether there is an advantage in treating BP by absolute risk is unlikely to be performed, because of the very large sample size and very long follow-up time required, particularly in patients at low risk. Therefore, reanalysis of the early placebo-controlled trials seems to be the most feasible approach for assessing the effects of delayed versus early drug treatment in individuals with varying CVD risk together and elevated BP.

Second, the estimation of HDLc from the 1980s national survey may alter the CVD risk score, but we do not believe this method greatly affected the risk stratification because a 0.4 difference in the HDL estimate only results in a 0.01 difference in CVD risk score. Furthermore, no association between HDLc and BP has been observed. ${ }^{33} 34$ The sensitivity analysis using Globorisk score ${ }^{29}$ without HDLc showed similar results as our main analysis. Although the ARR is no longer statistically significant, this result is likely due to the smaller sample size and subsequent number of events. In conclusion, the sensitivity analysis supports our main analysis.

Third, the paucity of trial endpoints in each CVD risk group prevented us from comparing the effects in some specific outcomes with respect to stroke and deaths from CVD. In addition, approximately one-third of the participants prematurely stopped randomised drug treatment. However, this pattern likely reflects the typical situation to occur in actual clinical practice, and this analysis is conducted on an intention-to-treat basis, so any difference in the estimate of treatment effect due to non-adherence is deliberately retained. Most participants were followed throughout the trial, except those with an unknown reason for stopping: loss to follow-up $(7.2 \%)$. An analysis with further adjustment by variable 
'premature stopped study treatment' did not substantially change our findings, except effects on stroke in general population became statistically significant $(0.55,95 \% \mathrm{CI}$ 0.30 to $0.99, \mathrm{P}=0.05)$. This is because non-adherence is balanced between the allocated treatment groups.

In conclusion, our research has demonstrated that drug treatment in patients with elevated BP is best directed to those at high risk of incident CVD events. This reinforces the guidelines recommendation to treat based on absolute (or global) CVD risk, rather than according to BP thresholds alone. ${ }^{5-9}$

Acknowledgements In the current study, the researchers gratefully acknowledge the RACGP Foundation and Therapeutic Guidelines Ltd for their support of this project.

Contributors MRN is responsible for the study conception and data archive from the Australian Data Archive. CLBH performed the analysis and drafted the manuscript. MB, CMR and JD provided substantial support on statistical analyses. All authors made great contribution to the interpretation of data, critically revised the manuscript and approved the final version.

Funding The ANBP was supported by the National Health and Medical Research Council of Australia, the Life Insurance Medical Research Fund of Australia and New Zealand, the Victorian Government, the Clive and Vera Ramaciotti Foundations and the Raine Medical Research Foundation of Western Australia.

Competing interests CLBH is a PhD candidate at Menzies Institute for Medical Research; she has received a PhD scholarship from Merle Weaver Postgraduate Scholarship. JD is supported by National Health and Medical Research Council Screening and Test Evaluation Program Grant 633003. CMR is supported by a National Health and Medical Research Council Senior Research Fellowship (1045862). MRN has in the last 5 years served on an advisory board for AMGEN.

Patient consent Detail has been removed from this case description/these case descriptions to ensure anonymity. The editors and reviewers have seen the detailed information available and are satisfied that the information backs up the case the authors are making.

Ethics approval This study was approved by the Tasmanian Health and Medical Human Research Ethics Committee (H0015252).

Provenance and peer review Not commissioned; externally peer reviewed.

Data sharing statement № additional data are available.

Open Access This is an Open Access article distributed in accordance with the Creative Commons Attribution Non Commercial (CC BY-NC 4.0) license, which permits others to distribute, remix, adapt, build upon this work non-commercially, and license their derivative works on different terms, provided the original work is properly cited and the use is non-commercial. See: http://creativecommons.org/ licenses/by-nc/4.0/

(C) Article author(s) (or their employer(s) unless otherwise stated in the text of the article) 2018. All rights reserved. No commercial use is permitted unless otherwise expressly granted.

\section{REFERENCES}

1. World Health Organisation. The top 10 causes of death. http://www. who.int/mediacentre/factsheets/fs310/en/ (accessed 13 Nov 2015).

2. Australian Bureau of Statistics. Causes of death, Australia, 2013. http://www.abs.gov.au/ausstats/abs@.nsf/Lookup/by\%20Subject/ 3303.0 2013 Main\%20Features Leading\%20Causes\%20of\% 20Death 10001 (accessed 30 Nov 2016).

3. Australia Bureau of Statistics. Causes of death, Australia, 2012. http://www.abs.gov.au/ausstats/abs@.nsf/Lookup/3303.0main+ features100012012 (accessed 13 Nov 2015).

4. World Health Organisation (WHO). A global brief on hypertension silent killer, global public health crissis. http://www.thehealthwell.info/ node/466541 (accessed 13 Nov 2015).

5. National Vascular Disease Prevention Allianace. Guidelines for the management of absolute cardiovascular disease risk. http://www. cvdcheck.org.au/index.php?option=com_content\&view=article\&id= 47\&ltemid=27. Updated (accessed 13 Nov 2015).
6. Task Force for the management of arterial hypertension of the European Society of HypertensionTask Force for the management of arterial hypertension of the European Society of Cardiology. 2013 ESH/ESC guidelines for the management of arterial hypertension. Blood Press 2013;22:193-278.

7. Piepoli MF, Hoes AW, Agewall S, et al. 2016 European Guidelines on cardiovascular disease prevention in clinical practice: the sixth joint task force of the European Society of Cardiology and Other Societies on Cardiovascular Disease Prevention in Clinical Practice (constituted by representatives of 10 societies and by invited experts) Developed with the special contribution of the European Association for Cardiovascular Prevention \& Rehabilitation (EACPR). Eur Heart $J$ 2016;37:2315-81.

8. Krause T, Lovibond K, Caulfield M, et al. Management of hypertension: summary of NICE guidance. BMJ 2011;343:d4891.

9. Dasgupta K, Quinn RR, Zarnke KB, et al. The 2014 Canadian Hypertension Education Program recommendations for blood pressure measurement, diagnosis, assessment of risk, prevention, and treatment of hypertension. Can J Cardiol 2014;30:485-501.

10. Diao D, Wright J, Cundiff $D$, et al. Pharmacotherapy for mild hypertension. Cochrane Database Syst Rev 2012;15:CD006742.

11. James PA, Oparil S, Carter BL, et al. 2014 evidence-based guideline for the management of high blood pressure in adults: report from the panel members appointed to the Eighth Joint National Committee (JNC 8). JAMA 20142014;311:507-20.

12. Whitworth JA. World Health Organization, International Society of Hypertension Writing Group. 2003 World Health Organization (WHO)/International Society of Hypertension (ISH) statement on management of hypertension. J Hypertens 2003;21:1983-92.

13. Weber MA, Schiffrin EL, White WB, et al. Clinical practice guidelines for the management of hypertension in the community. $J$ Clin Hypertens 2014;16:14-26.

14. Chobanian AV, Bakris GL, Black HR, et al. Seventh report of the joint national committee on prevention, detection, evaluation, and treatment of high blood pressure. Hypertension 2003;42:1206-52.

15. Mitka M. Groups spar over new hypertension guidelines. JAMA 2014;311:663-4.

16. Guallar E, Laine C. Controversy over clinical guidelines: listen to the evidence, not the noise. Ann Intern Med 2014;160:361-2.

17. Wright JT, Fine LJ, Lackland DT, et al. Evidence supporting a systolic blood pressure goal of less than $150 \mathrm{~mm} \mathrm{Hg}$ in patients aged 60 years or older: the minority view. Ann Intern Med 2014;160:499-503.

18. Zanchetti A. Bottom blood pressure or bottom cardiovascular risk? How far can cardiovascular risk be reduced? J Hypertens 2009;27:1509-20.

19. Wright JT, Williamson JD, Whelton PK, et al. A randomized trial of intensive versus standard blood-pressure control. N Engl J Med 2015;373:2103-16.

20. New Zealand Guidelines Group. The assessment and management of cardiovascular risk. Wellington: New Zealand Guidelines Group. http://www.health.govt.nz/publication/assessment-andmanagement-cardiovascular-risk (accessed 13 Nov 2015).

21. Morales Salinas A, Coca A, Olsen MH, et al. Clinical perspective on antihypertensive drug treatment in adults with grade 1 hypertension and low-to-moderate cardiovascular risk: an international expert consultation. Curr Probl Cardiol 2017:42:198-225.

22. Thomopoulos C, Parati G, Zanchetti A. Effects of blood pressure lowering on outcome incidence in hypertension: 2. Effects at different baseline and achieved blood pressure levels--overview and metaanalyses of randomized trials. $J$ Hypertens 2014;32:2296-304.

23. Lonn EM, Bosch J, López-Jaramillo P, et al. Blood-pressure lowering in intermediate-risk persons without cardiovascular disease. $N$ Engl J Med 2016;374:2009-20.

24. Sundström J, Arima H, Jackson R, et al. Effects of blood pressure reduction in mild hypertension: a systematic review and metaanalysis. Ann Intern Med 2015;162:184-91.

25. Blood Pressure Lowering Treatment Trialists' Collaboration. Blood pressure-lowering treatment based on cardiovascular risk: a metaanalysis of individual patient data. Lancet 2014;384:591-8.

26. The Australian therapeutic trial in mild hypertension. Report by the management committee. Lancet 1980;1:1261-7.

27. Anderson KM, Odell PM, Wilson PW, et al. Cardiovascular disease risk profiles. Am Heart J 1991;121:293-8.

28. Bennett SA, Magnus P. Trends in cardiovascular risk factors in Australia. Results from the National heart foundation's risk factor prevalence study, 1980-1989. Med J Aust 1994;161:519-27.

29. Hajifathalian K, Ueda P, Lu Y, et al. A novel risk score to predict cardiovascular disease risk in national populations (Globorisk): a 
pooled analysis of prospective cohorts and health examination surveys. Lancet Diabetes Endocrinol 2015;3:339-55.

30. Bender R, Kromp M, Kiefer C, et al. Absolute risks rather than incidence rates should be used to estimate the number needed to treat from time-to-event data. J Clin Epidemiol 2013;66:1038-44.

31. Zomer E, Owen A, Magliano DJ, et al. Validation of two Framingham cardiovascular risk prediction algorithms in an Australian population: the 'old' versus the 'new' Framingham equation. Eur J Cardiovasc Prev Rehabil 2011;18:115-20.
32. Hayward RA, Kent DM, Vijan S, et al. Multivariable risk prediction can greatly enhance the statistical power of clinical trial subgroup analysis. BMC Med Res Methodol 2006;6:1.

33. Hughes K, Leong WP, Sothy SP, et al. Relationships between cigarette smoking, blood pressure and serum lipids in the Singapore general population. Int J Epidemiol 1993;22:637-43.

34. Catalano M, Aronica A, Carzaniga G, et al. Serum lipids and apolipoproteins in patients with essential hypertension. Atherosclerosis 1991;87:17-22. 